\title{
SOME BANACH ALGEBRAS
}

EARL A. CODDINGTON

We define a class of commutative regular Banach algebras which contain simple examples of such algebras which are not self-adjoint. Conditions are given for the validity of the general Wiener theorem, and the denseness of certain translates. We use the Banach algebra terminology and results as presented in [1].

1. The Banach algebra $A$. Let $L^{2}(S)$ be the set of all complexvalued measurable functions, on a measure space $S$, whose magnitudes are square summable. We consider $L^{2}(S)$ as a Hilbert space, and assume the existence of a countable complete orthonormal set $\left\{\phi_{k}\right\}$ in $L^{2}(S)$, which has the additional property that $\phi_{k} \in L^{\infty}(S)$ $\cap L^{1}(S)$ for all $k$. For the given $\left\{\phi_{k}\right\}$ let $\left\{\nu_{k}\right\}$ be any sequence of nonzero complex numbers satisfying the condition

$$
\sum\left\|\phi_{k}\right\|_{\infty}^{2}\left\|\phi_{k}\right\|_{1}\left|\nu_{k}\right| \leqq 1,
$$

where $\left\|\phi_{k}\right\|_{\infty},\left\|\phi_{k}\right\|_{1}$ denote the norms of $\phi_{k}$ in $L^{\infty}(S)$ and $L^{1}(S)$ respectively. If $f, g \in L^{1}(S)$ we define the product $f * g$ by $^{1}$

$$
f * g=\sum\left(f, \phi_{k}\right)\left(g, \phi_{k}\right) \nu_{k}^{c} \phi_{k} .
$$

Here $\left(f, \phi_{k}\right)=\int_{S} f \phi_{k}^{c}$, and in general $(f, \alpha)=\int_{S} f \alpha^{c}$ in case $f \in L^{1}(S)$, $\alpha \in L^{\infty}(S)$, or in case $f, \alpha \in L^{2}(S)$. By (1), the series converges in $L^{1}(S)$, and indeed

$$
\|f * g\|_{1} \leqq\|f\|_{1}\|g\|_{1} \quad\left(f, g \in L^{1}(S)\right) .
$$

We have the following result:

$L^{1}(S)$ with * as multiplication is a commutative Banach algebra $A$ which is regular.

Proof. From (2) it follows that $A$ is closed under multiplication and satisfies the norm requirement for the product. The definition of the product clearly shows that the commutative and associative laws for multiplication are valid. Thus $A$ is a commutative Banach algebra.

It remains to prove that $A$ is regular, and for this we determine the regular maximal ideal space $\mathfrak{T}$ for $A$. The points $M$ of $\mathfrak{T}$ are in a one-to-one correspondence with the nonzero continuous homomorphisms of $A$ onto the complex numbers. Let $l_{M}$ be such a homo-

Presented to the Society, April 28, 1956; received by the editors March 5, 1956.

${ }^{1}$ For any $a, a^{c}$ denotes the complex conjugate of $a$. 
morphism corresponding to $M \in \mathscr{T}$. Then $l_{M}\left(\phi_{k}\right)$ cannot be zero for all $k$, for if the contrary is true, we have for any $f, g \in L^{1}(S)$,

$$
l_{M}(f) l_{M}(g)=l_{M}(f * g)=\sum\left(f, \phi_{k}\right)\left(g, \phi_{k}\right) \nu_{k}^{c} l_{M}\left(\phi_{k}\right)=0 .
$$

Since $l_{M}$ can not be identically zero, this gives a contradiction. Let $k$ be such that $l_{M}\left(\phi_{k}\right) \neq 0$. Then $l_{M}(f) l_{M}\left(\phi_{k}\right)=l_{M}\left(f * \phi_{k}\right)=\left(f, \phi_{k}\right) \nu_{k}^{c} l_{M}\left(\phi_{k}\right)$, or $l_{M}(f)=\left(f, \nu_{k} \phi_{k}\right)$. There is exactly one $k$ such that $l_{M}\left(\phi_{k}\right) \neq 0$, for $l_{M}\left(\phi_{j}\right)=\left(\phi_{j}, \nu_{k} \phi_{k}\right)=0$, if $j \neq k$. Thus corresponding to $M \in \mathfrak{T} C$ there is a unique integer $k$ such that $l_{M}(f)=\left(f, \nu_{k} \phi_{k}\right)$. Conversely every $\nu_{k} \phi_{k}$ generates in this way a continuous homomorphism onto the complex numbers, and the $f \in A$ satisfying $\left(f, \nu_{k} \phi_{k}\right)=0$ form a regular maximal ideal $M$. Therefore $\mathbb{T}$ can be identified with the integers $M \leftrightarrow k$.

For any $f \in A$ let $\hat{f}$ be the complex-valued function defined on $\mathfrak{T C}$ by $\hat{f}(M)=\hat{f}(k)=\left(f, \nu_{k} \phi_{k}\right)$. Then $A$ is said to be regular if, given any closed set $C$ in $\mathscr{T}$ and point $M_{0}$ not in $C$, there exists an $f \in A$ such that $\hat{f}(M)=0$ on $C, \hat{f}\left(M_{0}\right) \neq 0$. For our $A$ let $C$ be any set in $\mathscr{T}$ and $M_{0}$ a point not in $C$ determined by the integer $k$. Then $\phi_{k} \in A$ has the property that $\widehat{\phi}_{k}(j)=\delta_{j k} v_{j}^{c}$, thus showing that $A$ is regular.

The algebra $A$ is semi-simple (the map $f \rightarrow \hat{f}$ is one-to-one) if and only if $\left(f, \phi_{k}\right)=0$ for all $k$ implies $f=0$ almost everywhere. Suppose $A$ is semi-simple. If $f \in A$, and $\hat{f}$ vanishes outside a compact set, then $\left(f, \phi_{k}\right)=0$ for $|k|>N$, for some positive integer $N$. Thus

$$
f=\sum_{|k| \leq N}\left(f, \phi_{k}\right) \phi_{k}
$$

since $\left(f-\sum_{|k| \leqslant N}\left(f, \phi_{k}\right) \phi_{k}, \phi_{j}\right)=0$ for all $j$. It follows that the set of elements $f \in A$, such that $\hat{f}$ vanishes outside a compact set, is dense in $A$ if and only if the sequence $\left\{\phi_{k}\right\}$ is dense in $A$. The general Wiener Tauberian theorem (in the form of the Corollary, p. 85, [1]) then takes the following form.

Suppose the sequence $\left\{\phi_{k}\right\}$, which defines the algebra $A$, satisfies the two conditions:

(a) $\left(f, \phi_{k}\right)=0$, for $f \in L^{1}(S)$ and all $k$, implies $f=0$ almost everywhere,

(b) $\left\{\phi_{k}\right\}$ is dense in $L^{1}(S)$.

Then every proper closed ideal is included in a regular maximal ideal.

2. Some examples. A commutative Banach algebra $A$ is said to be self-adjoint if for every $f \in A$ there exists a $g \in A$ such that $\hat{g}=\hat{f}^{c}$. We give an example of a choice of $\left\{\phi_{k}\right\}$ and $\left\{\nu_{k}\right\}$ which lead to an algebra which is regular, but not self-adjoint. Let $S$ be the real interval $-\pi \leqq x \leqq \pi$, and $\phi_{k}=(2 \pi)^{-1 / 2} e^{-i k x}, k=0, \pm 1, \pm 2, \cdots$. Clearly $\phi_{k} \in L^{\infty}(S) \cap L^{1}(S) \cap L^{2}(S)$. The condition for self-adjointness is that for each $f \in L^{1}(S)$ there exists a $g \in L^{1}(S)$ such that $g(k)=\left(g, \nu_{k} \phi_{k}\right)$ 
$=\left(f, \nu_{k} \phi_{k}\right)^{c}$. If $\nu_{k}=\left|\nu_{k}\right| e^{i \theta_{k}}$, where $0 \leqq \theta_{k}<2 \pi$, this condition becomes $\left(g, \phi_{k}\right)=e^{2 i \theta_{k}}\left(f, \phi_{k}\right)^{c}$. Let $f^{-}(x)=[f(-x)]^{c}$. Clearly $f^{-} \in L^{1}(S)$ if and only if $f \in L^{1}(S)$, and $\left(f, \phi_{k}\right)^{c}=\left(f^{-}, \phi_{k}\right)$. Thus $L^{1}(S)$ is self-adjoint if and only if, given any $f^{-} \in L^{1}(S)$, there exists a $g \in L^{1}(S)$ such that

$$
\left(g, \phi_{k}\right)=e^{2 i \theta_{k}}\left(f^{-}, \phi_{k}\right) \quad(k=0, \pm 1, \cdots) .
$$

Now the function $f^{-}$defined by

$$
f^{-}(x)=\sum_{k=1}^{\infty} k^{-1 / 4} e^{i k x}
$$

is in $L^{1}(-\pi, \pi)$, but a sequence of \pm signs exist for which

$$
\sum_{k=1}^{\infty} \pm k^{-1 / 4} e^{i k x}
$$

is not the Fourier series of any function in $L^{1}(-\pi, \pi)$ (see [2, p. 212]). If we choose the $\theta_{k}$ so that $e^{2 i \theta k}$ gives such a sequence of signs, we see that $A$ will not be self-adjoint. Note that this $A$ is semi-simple and satisfies the conditions (a) and (b) for the general Wiener theorem.

Further examples are obtained by letting the $\phi_{k}$ be the orthonormal eigenfunctions of a self-adjoint ordinary differential operator on a finite closed interval $S: a \leqq x \leqq b$. The $\phi_{k}$ are continuous, and it is known that both (a) and (b) are valid.

Certain singular self-adjoint differential operators have a pure point spectrum. For example, the problem $-u^{\prime \prime}+q(x) u=\lambda u$, on $S$ : $-\infty<x<\infty$, is self-adjoint and has a pure point spectrum if $q$ is real-valued, continuous, and $q(x) \rightarrow \infty$ as $x \rightarrow \pm \infty$. It is known that in this case the orthonormal eigenfunctions $\phi_{k}$ are in $L^{\infty}(S) \cap L^{1}(S)$ $\cap L^{2}(S)$. For the particular case where $q(x)=x^{2}$ the $\phi_{k}$ are the Hermite functions, and the conditions (a) and (b) are valid.

3. The denseness of certain translates. For each $y \in S$ let $T_{y}$ be defined by

$$
T_{y} f(x)=\sum\left(f, \phi_{k}\right) \nu_{k}^{c}\left[\phi_{k}(y)\right]^{c} \phi_{k} .
$$

From (1) it follows that $\left\|T_{v} f\right\|_{1} \leqq\|f\|_{1}$ for every $f \in L^{1}(S)$, and an equivalent definition of $f * g$ is

$$
f * g(x)=\int_{S} T_{y} f(x) g(y) d y .
$$

We call $T_{y} f$ the translate of $f$ by $y$. Let $\mathfrak{T}_{f}$, for a given $f \in L^{1}(S)$, be the set of all finite linear combinations, with complex coefficients, of 
translates of $f$. It does not appear obvious that the closure of $\mathfrak{I}_{\boldsymbol{f}}$ is an ideal in $A$, in case $A$ satisfies (a) and (b). Nevertheless it is easy to show directly the following result:

Suppose $\left\{\phi_{k}\right\}$ is dense in $A$, and $f \in A$ is such that $\left(f, \phi_{k}\right) \neq 0$ for any $k$. Then $\mathfrak{T}_{f}$ is dense in $A$.

Proof. By the Hahn-Banach theorem $\mathfrak{T}_{f}$ is dense in $A$ if and only if the only bounded linear functional $l$ which satisfies $l(g)=0$ for all $g \in \mathfrak{T}_{f}$ is the identically zero one. Suppose $l(g)=0$ for all $g \in \mathfrak{T}_{f}$. In particular $l\left(T_{y} f\right)=0$ for all $y \in S$. Thus, using the definition (3),

$$
\sum\left(f, \phi_{k}\right) \nu_{k}^{c}\left[\phi_{k}(y)\right]^{c} l\left(\phi_{k}\right)=0 \quad(y \in S),
$$

where the series converges in $L^{\infty}(S)$ by (1). Since $\phi_{j} \in L^{1}(S)$ we have $0=\left(\phi_{j}, 0\right)=\left(f, \phi_{j}\right) \nu_{j}^{c} l\left(\phi_{j}\right)$, and, since $\left(f, \phi_{j}\right) \nu_{j}^{c} \neq 0$ for any $j$, this implies $l\left(\phi_{j}\right)=0$ for all $j$. This in turn implies that $l$ is the identically zero linear functional, since the $\phi_{j}$ are dense in $A$.

\section{REFERENCES}

1. L. H. Loomis, An introduction to abstract harmonic analysis, New York, 1953.

2. A. Zygmund, Trigonometrical series, Warsaw, 1935.

Mathematics Institute, University of Copenhagen and University of California, Los ANGeles 